(RESEARCH ARTICLE)

\title{
Respiratory tract, its implication, and the mathematical model for the determination of the radius of an alveolus.
}

\author{
Ikechukwu Iloh Udema * \\ ORCID: orcid.org/0000-0001-5662-4232
}

Department of Chemistry and Biochemistry, Research Division, Ude International Concepts LTD (RC: 862217), B. B. Agbor, Delta State, Nigeria. ORCID: orcid.org/0000-0001-5662-4232

Publication history: Received on 23 August 2020; revised on 24 September 2020; accepted on 26 September 2020

Article DOI: https://doi.org/10.30574/wjarr.2020.7.3.0321

\begin{abstract}
The flow rate of air has been investigated which showed that rate decreases per tract from one generation to other. However, the physiological implication of the tapering respiratory tract is not adequately elucidated and a noninvasive mathematical model is unknown for the computation of an alveolar radius. The formulation of mathematical models that can account for the effect of tapering airways and calculation of the radius of the alveolus at each generation of respiratory tracts are the objectives of the research. The method of research is theoretical and computational. Known radii of all respiratory tracts except the alveolus were substituted into derived equation for the calculation of alveolar radius. The rate of gas flow, in metres per second, decreases with increasing number of tracts from one generation to another. However, the sum of the distance covered per unit time increases from one generation to another. The alveolar radius for an average adult lung is $\approx 1.163 \pm 0.080$ (SD) exp $(-4)$ metre ( $\mathrm{df}=22$ and coefficient of variation is $\approx 6.879$ ). Older adults have longer alveolar radius in male and female subjects. Although the rate of gas flow in metres per second increases for awhile and then decreases, the total distance covered per unit time is increasingly high making the delivery of gas to the alveoli very rapid. The derived equations could be used for the computation of alveolar radius. There are gender, demographically and racially based differences in the radius of alveolus.
\end{abstract}

Keywords: Respiratory tract; Radius of alveolus; Rate of gas flow in volume per cross-sectional area per unit time; Implication of tapering diameter of respiratory tract.

\section{Introduction}

Researches in the past had focused on the determination of the radius of respiratory tract. The approaches need the use of more than one hi-tech equipment and cadaver. Where human subject is the case, the concern for safety dominates, hence the desire for and the use of non-invasive methods [1, 2]. Leong et al. [2] employed non-invasive phase contrast $\mathrm{X}$-ray (PCX) imaging technique to measure the size and population of alveoli in situ while Kjærgaard et al. [1] employed such methods for the determination of nasal cavity structure and air flow. In this research however, it is strongly believed that the radius of an alveolus can be determined mathematically and computationally in a non-invasive manner for subject in good health or ill health.

While the tapering diameter of the respiratory tracts towards the vasculature is well known, its implication does not seem to be taken into account in human respiration. To this end, Brown and Escombe [3], showed that pipe of narrower diameter transport $\mathrm{CO}_{2}$ at a faster rate measured in volume per unit cross sectional area per unit time than pipes of

\footnotetext{
${ }^{*}$ Corresponding author: Ikechukwu Iloh Udema

Department of Chemistry and Biochemistry, Research Division, Ude International Concepts LTD (RC: 862217), B. B. Agbor, Delta State, Nigeria.
}

Copyright (C) 2020 Author(s) retain the copyright of this article. This article is published under the terms of the Creative Commons Attribution Liscense 4.0. 
wider diameter. This has positive implication for the passive diffusion of gases through the stomata as well as through the respiratory tracts. The theory of gas flow through respiratory tracts has also been postulated, as exemplified in the work of Iorungwa [4]. Though not too familiar, the flow system in the exchange of gas between blood and alveolar has been described according to the continuity and Navier-Stokes equations in the cylindrical coordinate [4].

The need for a noninvasive method for the determination of alveolar radius, in the face of current global pandemic, cannot be overemphasised. The treatment involving appropriate therapeutics also requires a means of prognoses and evaluation of the effectiveness and efficacy of treatments administered by the medics and paramedics at the frontline. It is on account of this that this research is conducted with the objectives of formulating mathematical models suitable to the lung that can account for the effect of tapering airways and to calculate the radius of the alveolus at each generation of respiratory tracts.

\section{Theory}

There is a need to derive a simple equation that can be used to quantify the effect of tapering diameter of respiratory tract. This should enhance the elucidation of the value or importance of natural anatomical structure of the respiratory tree. This elucidation at theoretical level begins with Eq. (1) [5] below.

$$
u=\sqrt[3]{\frac{v^{2} h}{m \phi \lambda}}
$$

Where, $\lambda$ is the de Broglie wavelength of the gas and $\phi$ is a positive integer $\geq 1 ; v, m$ and $h$ are the root mean square velocity of the gas, mass of a molecule of the gas, and Planck constant respectively. This is the $1^{\text {st }}$ principle basis for the effect of tapering diameter or short diameter of a conduit pipe as elucidated elsewhere [5]. Larger values of $\phi$ leads to higher entropic term or randomness, reducing as such the rate of delivery of needed air to the alveolar end. If $\phi \rightarrow 1$, which is extremely rare, the air molecules will be delivered at a velocity equal to $v$.

It is known in the literature that certain respiratory parameter may be dependent on or could be a function of certain anatomical feature. For instance Schriever et al [6] observed that the "relationship between various anatomical measures is, however, well documented. For example, a tall individual with long legs also tends to have long arms". Therefore, on account of this the researcher examined the relationship between intranasal volume and size of the nares with the aim of using the area of the nostril opening as a potentially simple predictor of total intranasal volume. This, therefore, informed the desire in this research to compute the radius of the alveolus on the assumption that it may depend on the radius of other respiratory tract.

\subsection{Determination of the intra-luminal rate of gas flow in volume per unit cross-sectional area per unit time.}

Taking Etract and $v_{\text {tract }}$ as the number and volume of respiratory tract respectively in a given generation, the rate of gas diffusion $R_{\mathrm{v}}$, in unit of volume per unit time, into the "respiration tree" can be related to the time $t$ it may take to transport by diffusion a volume of gas equal to the volume of a unit tract in a given generation as follows.

$$
\nu_{\text {tract }} / t=R_{\mathrm{v}} / \varepsilon_{\text {tract }}
$$

Therefore, $t=v_{\text {tract }} \varepsilon_{\text {tract }} / R_{\mathrm{v}}$, and given that the volume of gas equal to a volume $\left(R_{\text {tract }}\right)$ per a unit of a given generation is to be delivered into the lung generally, then, the rate of gas flow (or diffusion), $R_{\mathrm{A}}$ in volume per cross-sectional area per unit time is given respectively for tube-like tract and alveolus as follows:

$$
R_{A}=\frac{R_{\text {tract }} R_{\mathrm{V}}}{\pi r_{\text {tract }}^{2} V_{\text {tract }} \varepsilon_{\text {tract }}}
$$

However, it is likely that $v_{\text {tract }}=R_{\text {tract }}$ such that, Eq. (3) becomes

$$
R_{A}=\frac{R_{\mathrm{V}}}{\pi r_{\text {tract }}^{2} \varepsilon_{\text {tract }}}
$$

If sphericity is assumed for the alveolus, then

$$
R_{A}=\frac{R_{\mathrm{V}}}{4 \pi r_{\mathrm{alv}}^{2} \varepsilon_{\text {tract }}}
$$


Figure 1 [7] illustrates the various tracts in each generation of the respiratory tree. While the number of respiratory tract $\left(N_{\text {tract }}\right)$ in each generation except $24^{\text {th }}$ can be computed with the equation, $N_{\text {tract }}=2^{\mathrm{z}}$ (where $\mathrm{Z}$ is the generation number), the diameters and length of tracts were adopted from the work of Weiber [8] as compiled by Gehr [9].

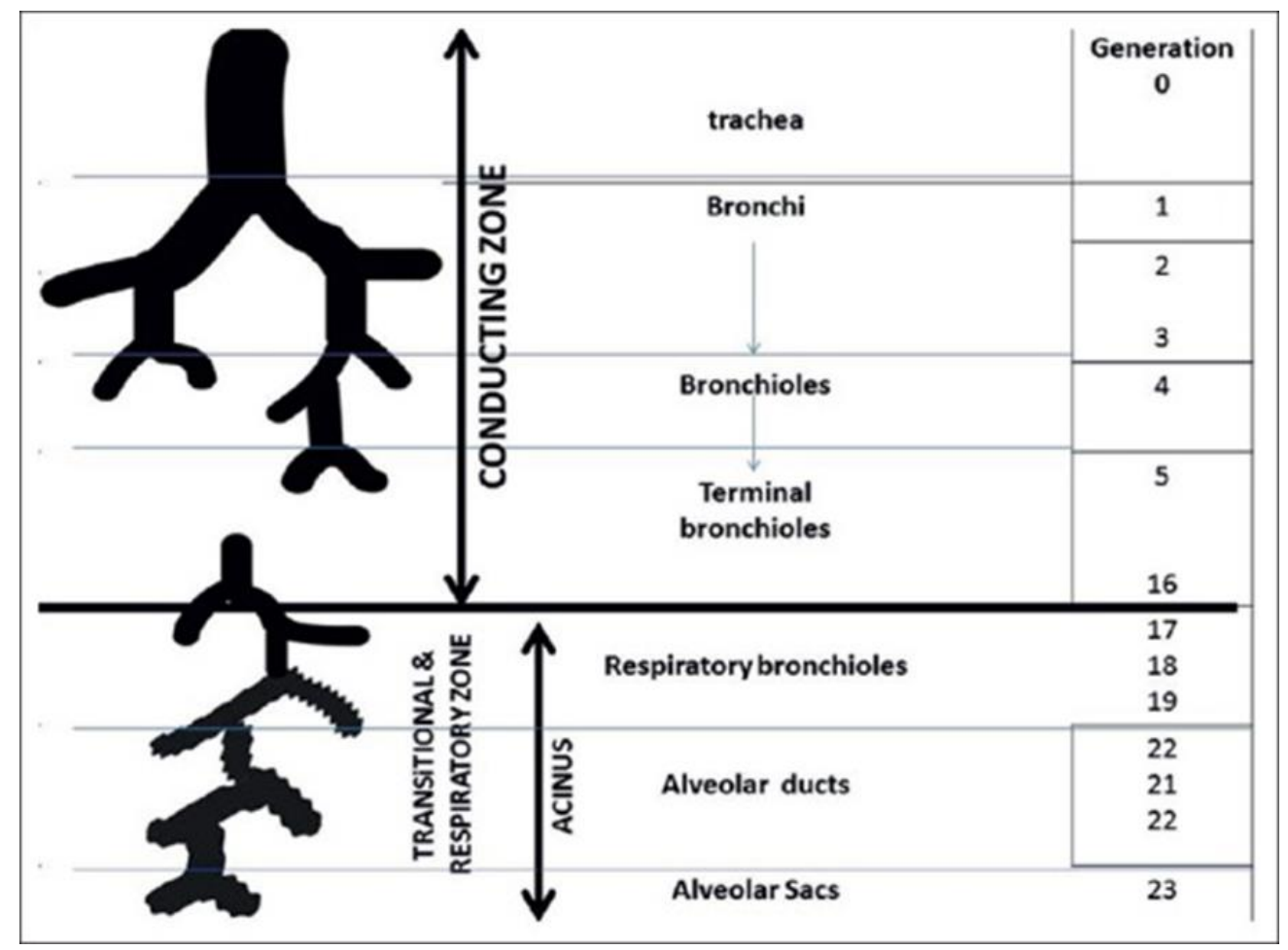

Figure 1 Schematic diagram of a respiratory tree. Adopted from the work of Patwa and Shah [7]

\subsection{Derivation of the equation for the calculation of alveolar radius}

Previous research [10] produced a mathematical equation for the determination of the number of alveoli in a human lung. The equation, Eq. (6) below, have to be modified so as to be adoptable for the determination by computation, of the radius of respiratory alveolus.

$$
\varepsilon=\left(v d^{22}\right)^{1 / 15}\left(\frac{R_{\mathrm{V}}}{4 \pi}\right)^{3 / 5}\left(\frac{m}{h}\right)^{2 / 3} / r_{\infty}^{2}
$$

Where, ' $\varepsilon$ ', $v, d$, and $R_{\mathrm{v}}$ in its original definition [10], are the number of alveoli, root mean square velocity, width of the nares measured from one end to another as described by Zaidi et al [11] and rate of gas diffusion in unit of volume per unit time; $m, h$ and $r_{\infty}$ are the mass of a molecule of gas, Planck constant, and the radius of an alveolar. In order to determine the diameter (or radius) of an alveolus as a function of a definite respiratory tract radius, other than the alveolus, which is not easily accessible, Eq. (6) has to be modified by multiplying $R_{\mathrm{v}}$ by the factor, the volume of an alveolus divide by the volume of the cylindrical model of the tract such as any of the following: Trachea, bronchus (left or right), bronchiole etc. The equation is given below.

$$
\varepsilon_{\text {pipe }}=\sqrt[15]{v d_{\text {pipe }}^{22}}\left(\sqrt[5]{\left(\frac{R_{\mathrm{V}} V_{\text {alv }}}{\pi r_{\text {pipe }}^{2} l_{\text {pipe }}} \frac{1}{4 \pi}\right)}\right)^{3}\left(\sqrt[3]{\frac{m}{h}}\right)^{2} \frac{1}{r_{\text {pipe }}^{2}}
$$

Where, $\varepsilon_{\text {pipe, }} d_{\text {pipe, and }} R_{\mathrm{v}}$ are the number of a respiratory "conduit pipe" taken to be one (because in other generations of respiratory tract which excludes the zero generation where the trachea is placed, the average diameter of all the tracts in each generation is taken), the average diameter of a respiratory "conduit pipe" and the rate of diffusion of gas in unit of volume per unit time respectively; $r_{\text {pipe, }} V_{\text {alv, }}$ and $l_{\text {pipe }}$ are the radius of a respiratory "conduit pipe" the volume of an alveolus, and the length of a respiratory respectively. If a spherical model of an alveolus is taken, Eq. (7a), can be simplified to 


$$
\varepsilon_{\text {pipe }}=\sqrt[15]{v d_{\text {pipe }}^{22}}\left(\sqrt[5]{\left(\frac{R_{\mathrm{v}} r_{\text {alv }}^{3}}{3 \pi r_{\text {pipe }}^{2} l_{\text {pipe }}}\right)}\right)^{3}\left(\sqrt[3]{\frac{m}{h}}\right)^{2} \frac{1}{r_{\text {pipe }}^{2}}
$$

Where, $r_{\text {alv }}$ is the radius of an alveolus ( $r_{\infty}$ is replaced). By substituting $2 r_{\text {pipe }}$ into Eq. (7b) in place of $d_{\text {pipe, }}$ one obtains after rearrangement the equation below.

$$
\sqrt[5]{r_{\text {alv }}^{9}}=\frac{(3 \pi)^{3 / 5}}{\sqrt[15]{4194304 v}}\left(\sqrt[3]{\frac{h}{m_{\mathrm{g}}}}\right)^{2} \cdot\left(\sqrt[5]{\frac{l_{\text {pipe }}}{R_{\mathrm{v}}}}\right)^{3} \cdot \sqrt[15]{r_{\text {pipe }}^{26}} \cdot \varepsilon_{\text {pipe }}
$$

Where $m_{\mathrm{g}}$ is the mass of a gas molecule, air in this research given as ( $\%$ C. $\left.M_{\mathrm{C}}+\% 0 . M_{\mathrm{O}}+\% \mathrm{~N} . M_{\mathrm{N}} \ldots\right) / N_{\mathrm{A}} 100$ where $\%$ C, $\% \mathrm{O}$, and $\% \mathrm{~N}$ are the proportions in percentage of carbon (IV) oxide, molecular oxygen, and molecular nitrogen respectively; $M_{\mathrm{C}}, M_{\mathrm{O}}$, and $M_{\mathrm{N}}$ are the molar masses of carbon dioxide, oxygen, and nitrogen molecules respectively. The presence of rare gases is ignored. Rearrangement of Eq. (7c) gives

$$
r_{\text {alv }}=\sqrt[9]{\left(\frac{(3 \pi)^{3 / 5}}{\sqrt[15]{4194304}}\left(\sqrt[3]{\frac{h}{m_{\mathrm{g}}}}\right)^{2}\right)^{5}} \sqrt[9]{\sqrt[3]{\frac{r_{\text {pipe }}^{26}}{v}}\left(\frac{l_{\text {pipe }}}{R_{\mathrm{v}}}\right)^{3}}
$$

The parameter epipe is omitted in Eq. (7d) because it is one in zero generation of the respiratory tree (RT), the trachea in this case and in subsequent generations as explained earlier. Besides the equation is suitable where breathing at rest is the case. If the $1^{\text {st }}$ and higher generation of the RT is the case, Eq. (7d) needs further modification. Besides, in subsequent generations, the arithmetic mean of the diameters and lengths are to be taken such that $\varepsilon_{\text {pipe }}$ will always be

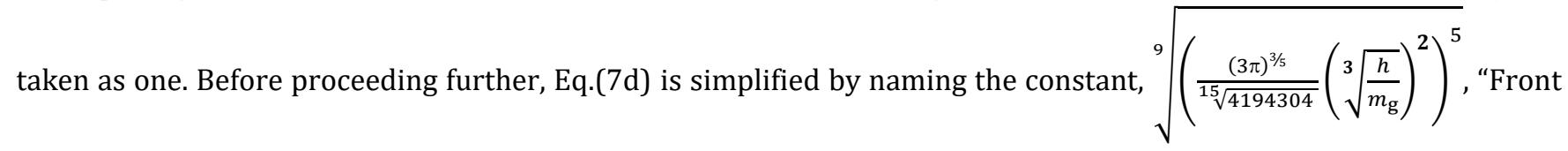
Liners Against Covid-19" (FAC-19) constant in recognition of the tremendous efforts of medics and paramedics in the battle against the pandemic and also in memory of those who lost their lives world-wide. The unit of FAC-19 constant is $\left(\mathrm{m}^{2} / \mathrm{s}\right)^{10 / 27}$. Taking the value of $m_{\mathrm{g}}$ to be $\sim 4.965 \exp (-26) \mathrm{g}$, the value of the constant is: $\sim 1.45532 \exp (-3)$ $\left(\mathrm{m}^{2} / \mathrm{s}\right)^{10 / 27}$.

If the $1^{\text {st }}$ or any other higher generation is in focus, Eq. (7c) needs to be multiplied by the factor given as ( $(z$ $\left.\left.l_{\text {trach }} r_{\text {trach }}^{2} / l_{\text {pipe }} r_{\text {pipe }}^{2} z\right)^{3 / 5}\right)^{5 / 9}$. Hence,

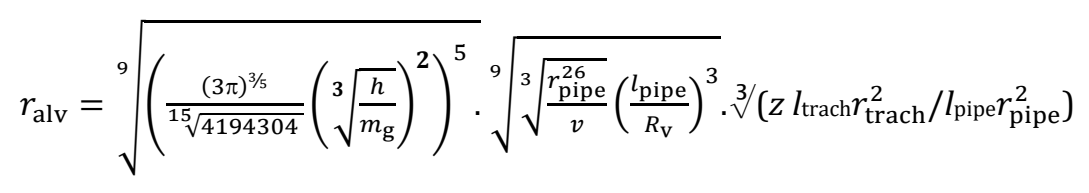

Where, $l_{\text {trach }}$ and $l_{\text {pipe }}$ mean the length of trachea and any other respiratory pipe respectively; the corresponding radii are $r_{\text {trach }}$ and $r_{\text {pipe }}$ respectively and, $z$ is any other generation other than zero. To make the equations less cumbersome, Eq. (7c) and Eq. (7e) can be re-written respectively as:

$$
\begin{aligned}
& r_{\text {alv }}=1.45532 \exp (-3) \sqrt[9]{\sqrt[3]{\frac{r_{\text {trach }}^{26}}{v}}\left(\frac{l_{\text {trach }}}{R_{\mathrm{v}}}\right)^{3}} \\
& r_{\text {alv }}=1.45532 \exp (-3) \sqrt[9]{\sqrt[3]{\frac{r_{\text {pipe }}^{26}}{v}}\left(\frac{l_{\text {pipe }}}{R_{\mathrm{v}}}\right)^{3}} \times \sqrt[3]{ }\left(z l_{\text {trach }} r_{\text {trach }}^{2} / l_{\text {pipe }} r_{\text {pipe }}^{2}\right)
\end{aligned}
$$

Equation (9a) can further be simplified to

$$
r_{\text {alv }}=1.45532 \exp (-3) \sqrt[3]{\frac{Z r_{\text {trach }}^{2}}{R_{\mathrm{v}}}} \times \sqrt[27]{\frac{r_{\text {pipe }}^{8}}{v}} .
$$


One can deduce that when $z=0$, the respiratory tract is the trachea and Eq. (8) is suitable for the calculation of the radius (or diameter) of the alveolus. On the other hand, if $z \geq 1$, Eq. (9) should be the case, for the calculation of the radius of the alveolus. Both equations are applicable for resting condition. It must be noted that if pure molecular oxygen is the gas, then the constant, christened FAC-19 constant should be $\sim 1.41919 \exp (-3)\left(\mathrm{m}^{2} / \mathrm{s}\right)^{10 / 27}$.

\section{Material and methods}

\subsection{Materials}

There was no any form of measurement.

\subsection{Methods}

The method is purely computational which entailed the substitution of relevant data into Eqs (8), and (9b).

\section{Results and discussion}

Any discussion or analysis related to gas flow through the respiratory tree requires existing pieces of information on the same issue in the literature. Successful flow of gas through the respiratory tract demands the effective exchange of gas between the gas "reservoir portion", the alveoli and the vasculature; inhaled gas diffuses from the alveolar end into the blood across the alveolar-capillary barrier and from the blood into the alveoli by well-established mechanism. There is implication arising from increasing number of tracts with subsequent generation, often associated with decreasing diameter of the tracts. Such implication is best discussed after a brief critique of information in the literature.

At rest, the mean flow velocity on inspiration is about $1 \mathrm{~m} / \mathrm{s}$ in the trachea and less than $1 \mathrm{~cm} / \mathrm{s}$ in the first-order respiratory bronchioles [12]. This is against the backdrop of Fick's law of diffusion which states that the rate of diffusion of a gas across a permeable membrane is determined by the chemical nature of the membrane itself, the surface area of the membrane, the partial pressure gradient of the gas across the membrane, and the thickness of the membrane (www.pathwaymedicine/Respiratory Physiology/Pulmonary gas exchange). Besides, the mean flow rate stated in $\mathrm{m} / \mathrm{s}$ if it is intended to mean units of volume per cross-sectional area of tract per second is $<$ the $40 \mathrm{~L} / \mathrm{min}$ minute ventilation test standard (for tests with a sinusoidal flow pattern) $100 \%$ of the time [13]. Therefore, the relevant gas exchange features of lung anatomy are the very large surface area $\left(70 \mathrm{~m}^{2}\right)$ and the thin diffusion barrier $(0.10 \mu \mathrm{m})$ between the blood and alveolar gas [14].

Table 1a Rate of gas flow in units of volume per unit time per cross-sectional area at each generation of the respiratory tree.

\begin{tabular}{|c|c|c|c|c|c|c|c|c|c|c|c|}
\hline$Z$ & $N_{\text {tube }}$ & $\begin{array}{l}u / \exp (-4) \\
\mathrm{m} / \mathrm{s}\end{array}$ & $z$ & $N_{\text {tube }}$ & $\begin{array}{l}u / \exp \\
(-4) \mathrm{m} / \mathrm{s}\end{array}$ & $z$ & $N_{\text {tube }}$ & $\begin{array}{l}\text { u/exp } \\
\left(-{ }^{2} \quad 4\right) \\
m / s\end{array}$ & $Z$ & $N_{\text {tube }}$ & $\begin{array}{l}u / \exp \\
(-4) \mathrm{m} / \mathrm{s}\end{array}$ \\
\hline 0 & 1 & 333.895* & 6 & 64 & 215.605 & 12 & 4096 & 29.265 & 18 & 262144 & 1.651 \\
\hline 1 & 2 & 363.416 & 7 & 128 & 159.768 & 13 & 8192 & 19.640 & 19 & 524288 & 0.934 \\
\hline 2 & 4 & 392.589 & 8 & 256 & 122.149 & 14 & 16384 & 12.058 & 20 & 1048576 & 0.509 \\
\hline 3 & 8 & 431.121 & 9 & 512 & 89.093 & 15 & 32768 & 7.579 & 21 & 2097152 & 0.279 \\
\hline 4 & 16 & 333.895* & 10 & 1024 & 62.513 & 16 & 65536 & 4.585 & 22 & 4194304 & 0.153 \\
\hline 5 & 32 & 275.974 & 11 & 2048 & 44.460 & 17 & 131072 & 2.830 & 23 & 8388608 & 0.077 \\
\hline 24 & $225^{\wedge} 6$ & \multicolumn{10}{|c|}{$1.923 \exp (-7) \mathrm{m} / \mathrm{s}$} \\
\hline
\end{tabular}

* indicates the fact that the rate $u$ at the two generations, 0 and 4 are the same, a coincidence that is not unlikely; 225 exp (+6) i.e. $225^{\wedge} 6$ is the number of alveoli that may have been mobilised based on the assumption that $3 / 4$ of lung volume was inflated for an average adult [8] and a radius of an alveoli $=1.25 \exp (-4) \mathrm{m}$ [18]. The symbols, $z, N_{\text {tube, }}$ and $u$ are the generation number, number of tubes or tracts, and rate in m/s respectively.

Meanwhile, the reported unit of flow rate, if there is no mistake, seems to be an overestimate, if flow rate per crosssectional area of trachea was the case, compared with the corrected minute respiratory volume expected from the product of tidal volume (500 mL) minus dead space air $(150 \mathrm{~mL})$ [15] and respiratory rate or frequency (12/min) which gives $\approx 0.275 \mathrm{~m} / \mathrm{s}$. With reference to the literature [16], a respiratory rate of 12 breaths /min and a tidal volume approximated as $10 \%$ of the subject's vital capacity had been used elsewhere [17]. The authors reported several flow rates ranging from 140-450 mL/s which is » $7.8 \exp (-5) \mathrm{m}^{3} / \mathrm{s}$ (computed using 12 breaths per minute and $10 \%$ of average vital capacity $((=5.4$ litre $)[17]-0.150$ litre $)$. 
Equations (3) and (4) were used for the determination of the rate (velocity) of gas flow in unit of volume per unit time per cross-sectional area of each respiratory tract. This gives the unit of velocity. This is after the work of Escombe and Brown [3] showed the effect of diameter on the rate of gas flow through cylindrical pipes in part and subsequent to the theory that explains why pipes of narrower aperture allow faster velocity of gas transit than wider aperture [5].

There is clear evidence that the volume of gas in a tract decreases with increase in the number of tracts in every generation so that the increasing value of $u$ (Table 1a) is not ad infinitum. There may not be a clear-cut reason why the coincidental equality in values of $u$ in zero and $4^{\text {th }}$ generations may not be applicable to any other generation either immediately before or after the $4^{\text {th }}$ generation; the closet answer falls in the realm of mathematics. The observed increases have the implication of accelerating the delivery despite the need for the avoidance of turbulence, perhaps, via the decrease to initial rate in zero generation. This may present a regulatory effect as an implication in part. This may enable the exchange of gas at the capillary-alveolar barrier to keep pace with the rate of gas supply or number of breath per unit time. As Hsia et al [12] observed, there appeared to be a decreasing trend in the velocity of gas flow measured in metres per second in the trachea and in centimetres per seconds in the $1^{\text {st }}$ order respiratory bronchioles. Unfortunately no mathematical equation was clearly indicated by the authors.

While there is continuous decrease in volume of gas flow per respiratory tract, resulting in decrease in $u$ beginning from the $5^{\text {th }}$ generation (Table $1 \mathrm{~b}$ ), despite decreasing trend in radii through the generations, what is more important is the total distance covered per unit time through all the tracts (bifurcations). Thus, the product of the number of tract per generation and $u$ gives an idea of the overall rate at which the gas is delivered to the alveoli. This is best illustrated in Table $1 \mathrm{~b}$. In zero generation containing the trachea the inspired gas passes through one tract, the same volume of gas passes through several tracts in other generation, an observation that seems trivial yet has important positive effect or implication. Apart from the effect of large surface area of the entire lung, the massive delivery of gas to the alveoli is better visualised in terms of total distance covered per unit time which is shortest with the trachea and longest with the total alveoli. The division of respiratory tract into several tracts in each generation also, enhances directionality and reduces randomness and consequently the entropic term as explained elsewhere [5].

Table 1b Result showing the effect of division of respiratory tree into several tracts in subsequent generation

\begin{tabular}{|c|c|c|c|c|c|c|c|c|c|c|c|}
\hline $\mathbf{Z}$ & $N_{\text {tube }}$ & $u / \mathrm{m} / \mathrm{s}$ & $Z$ & $N_{\text {tube }}$ & $u / \mathrm{m} / \mathrm{s}$ & $Z$ & $N$ tube & $u / \mathrm{m} / \mathrm{s}$ & $Z$ & $N_{\text {tube }}$ & $\begin{array}{l}u / \exp \\
(-4) \mathrm{m} / \mathrm{s}\end{array}$ \\
\hline 0 & 1 & 0.033 & 6 & 64 & 1.380 & 12 & 4096 & 11.987 & 18 & 262144 & 43.280 \\
\hline 1 & 2 & 0.073 & 7 & 128 & 2.045 & 13 & 8192 & 16.089 & 19 & 524288 & 48.968 \\
\hline 2 & 4 & 0.157 & 8 & 256 & 3.127 & 14 & 16384 & 19.756 & 20 & 1048576 & 53.373 \\
\hline 3 & 8 & 0.335 & 9 & 512 & 4.562 & 15 & 32768 & 24.835 & 21 & 2097152 & 58.511 \\
\hline 4 & 16 & 0.534 & 10 & 1024 & 6.401 & 16 & 65536 & 30.048 & 22 & 4194304 & 64.173 \\
\hline 5 & 32 & 0.883 & 11 & 2048 & 9.105 & 17 & 131072 & 37.093 & 23 & 8388608 & 129.185 \\
\hline 24 & $225^{\wedge} 6$ & 43.273 & & & & & & & & & \\
\hline
\end{tabular}

Table 2 The calculated value of the radius of alveolus in each generation of the respiratory tree and the average for an average adult lung.

\begin{tabular}{|c|c|c|c|c|c|c|c|c|c|c|c|}
\hline$Z$ & $N_{\text {tube }}$ & $\begin{array}{l}r_{\text {alv }} / \exp \\
(-4) \mathrm{m}\end{array}$ & $Z$ & $N_{\text {tube }}$ & $\begin{array}{l}r_{\text {alv }} / \exp \\
(-4) \mathrm{m}\end{array}$ & $Z$ & $N_{\text {tube }}$ & $\begin{array}{l}r_{\text {alv }} / \exp \\
(-4) \mathrm{m}\end{array}$ & $\boldsymbol{Z}$ & $N_{\text {tube }}$ & $\begin{array}{l}r_{\text {alv }} / \exp \\
(-4) \mathrm{m}\end{array}$ \\
\hline 0 & 1 & 1.200 & 6 & 64 & 1.369 & 12 & 4096 & 1.149 & 18 & 262144 & 1.088 \\
\hline 1 & 2 & 1.070 & 7 & 128 & 1.248 & 13 & 8192 & 1.130 & 19 & 524288 & 1.088 \\
\hline 2 & 4 & 1.202 & 8 & 256 & 1.225 & 14 & 16384 & 1.124 & 20 & 1048576 & 1.092 \\
\hline 3 & 8 & 1.225 & 9 & 512 & 1.205 & 15 & 32768 & 1.112 & 21 & 2097152 & 1.095 \\
\hline 4 & 16 & 1.264 & 10 & 1024 & 1.187 & 16 & 65536 & 1.105 & 22 & 4194304 & 1.097 \\
\hline 5 & 32 & 1.263 & 11 & 2048 & 1.230 & 17 & 131072 & 1.092 & 23 & 8388608 & 1.053 \\
\hline $\mathrm{Av}$ & \multicolumn{11}{|c|}{$\sim 1.163 \pm 0.080(\mathrm{SD}) \exp (-4)$ metres; $\mathrm{df}=22$} \\
\hline
\end{tabular}

The symbols, $z, N_{\text {tube, }}$ and $r_{\text {alv }}$ are the respiratory generation number, number of tubes, and radius of alveolus respectively. Coefficient of variation is $\approx 6.879$.

The data recorded in the work of Gehr [9] was adopted from 1963 text by Weibel [8] and employed in this research for an average adult. There was no indication as to whether or not the data is either for a female, male or both. Therefore, generalisation is limited. To be noted, however, is the fact that, the low coefficient of variation of the alveolar radius calculated as a function of the respiratory tract radii showed that the variability of the sample is low relative to the mean 
radius of the alveolus (Table 2). What needs to be emphasised is the procurement or generation of adequate and almost totally accurate data on the length and diameter of respiratory tracts against the backdrop of established race-based, gender-based, and genetically orchestrated differences in the magnitude of those parameters. The works of Breatnach et al [19], Zahedi-Nejad et al [20], and Lee et al [21] provide pieces of evidence on this issue.

Tables 3a and 3b contain results generated by exploring the data in the literature [22]. Each datum was substituted into relevant equation for the purpose of calculating the radius of alveolus. However, it is imperative to observe that different data on the radius and length of the respiratory tract abound in the literature. Information about the issue is pivotal for any computation. Breatnach et al [19] reported different values of the coronal and sagittal tracheal diameters in normal subjects, male and female, within different age brackets. Coronal and sagittal tracheal diameters were defined as the internal diameters of the tracheal air column as measured at a level $2 \mathrm{~cm}$ above the projected top of the aortic arch on posterior-anterior and lateral radiograph respectively [19]. Zhang et al [23] reported values equal to $10.78 \pm 1.32$ and $10.14 \pm 1.28 \mathrm{~cm}$ for the male and female subjects respectively among Chinese population. A value of $8.9 \mathrm{~cm}$ in length for a subject (biodata: 18 years, $1.75 \mathrm{~m}$ in height, and $70 \mathrm{~kg}$ in weight) was according to Gehr [9] reported by Phalen et al [24]. As such none of them can specifically give a definitive result if substituted into the equations. The values, $10.5 \pm$ 1.2 for male and $10.2 \pm 1.0 \mathrm{~cm}$ for female stretched cadavers were reported elsewhere [25]. The values at rest, according to Cinar et al [25] were shorter in length. The use of cadaver presents its own limitation in that the occurrence of rigor mortis requires the stretching of the cadaver trachea whose structural (or rather anatomical integrity) may differ widely from the healthy or sick (but non-terminal) subject. Stretching may not necessarily produce a length equal to the native normal length. In this regard and in recognition of earlier report Griscom and Wohl [22] observed that the measurements taken and reported in their research were generally larger than those found at autopsy (use of cadaver is implied in this case). Incidentally, $10 \mathrm{~cm}$ in length was recorded 40 years ago for the human trachea [18] besides 12 $\mathrm{cm}$ recorded in 1963 [9]. The value equal to $10 \mathrm{~cm}$ encourages one to adopt a range of 10 to $10.7 \mathrm{~cm}$.

Racially based differences is inevitable in line with the result by Zahedi-Nejad et al [20] which showed that, for the Iranians, the sagittal upper tracheal diameter for the males and females were 2.06 and 1.49 cm respectively. Without any reference to any geopolitical location (though the names of the authors seem to point in the direction of Asian continent) Lee et al [21] report the length and the anterior-posterior and transverse diameter of the left main bronchus (LMB) $2 \mathrm{~cm}$ below the carina as well as the length and both anterior-posterior and transverse diameters of mid-point of the right main bronchus for both male and female, with latter being shorter.

The radius of alveoli was calculated for each age-group. The calculation is based on Eq. (8) which expresses the radius of alveolus mainly as a function of the length and diameter of the respiratory tract. The results shown in Tables $3 a$ and $3 \mathrm{~b}$ are important because they cover several age-groups or brackets of different sexes. It is therefore, generally highly representative, though it may not cover all races. The data substituted into the equation were obtained from the work of Breatnach et al [19] and the arithmetic mean of the length was calculated from the data reported by Grisom and Wohl [22]. The radii calculated for the male, sagittally and coronally, were as expected, generally longer than for the female subjects. One needs to acknowledge the fact that 16 years of age may be described as non-adolescence in characteristics. Nevertheless, a rough estimate may be assumed for easier descriptive analysis and discussion. Thus, one sees as shown in Table 3a, an increasing trend in the radius with increasing values of the age range up to 40-49 years from coronal perspective, a decrease at 50-59 years, and a further increase to a peak value at maximum age bracket. This may in part be attributed to unequal population of subject employed for the investigation. However, there is a regular increase in radii with increasing age from sagittal perspective.

Table 3a The various values of alveolar radii for different male age-brackets as a function of trachea length and diameter.

\begin{tabular}{|l|l|l|l|}
\hline Age groups (years) & \multirow{2}{*}{ No. of Subjects } & Radius / exp (-4) m & Radius / exp (-4) m \\
\cline { 3 - 4 } & & Coronal perspective & Sagittal perspective \\
\hline $10-19$ & 26 & $1.089 \pm 0.213$ & $1.082 \pm 0.231$ \\
\hline $20-29$ & 81 & $1.372 \pm 0.159$ & $1.414 \pm 0.159$ \\
\hline $30-39$ & 72 & $1.376 \pm 0.158$ & $1.410 \pm 0.186$ \\
\hline $40-49$ & 69 & $1.396 \pm 0.178$ & $1.451 \pm 0.171$ \\
\hline $50-59$ & 80 & $1.375 \pm 0.178$ & $1.458 \pm 0.201$ \\
\hline $60-69$ & 71 & $1.396 \pm 0.113$ & $1.479 \pm 0.133$ \\
\hline $70-79$ & 31 & $1.410 \pm 0.171$ & $1.486 \pm 0.141$ \\
\hline
\end{tabular}

The grouping by age and the number of subjects and the diameter of the trachea are as adopted from the work of Breatnach et al [19]; the arithmetic means of the length, $10.67 \mathrm{~cm}$ and $12.4 \mathrm{~cm}$ were calculated from values adopted from the work of [22]. 
The calculated radii of alveolus for age bracket above 10-19 years for the male (Table 3a) are longer than the value 1.25 $\exp (-4)$ metres reported for resting subject in the literature [18, 26]. Balashazy et al [26] reports an average minimum alveolar radius $=1.165 \exp (-4)$ metres on the basis of a tidal volume (TV) equal to $750 \mathrm{~mL}$ per breath during resting condition and a value of $1.365 \exp (-4)$ metres during heavy exercise given a TV equal to $1923 \mathrm{~mL}$ per breath. It appears the longer radii observed in this research may be as a result of different demographic structure used by previous researchers. Genetic differences within and between races could be a predisposing factor. It could therefore, appear that longer radius can be observed for some subjects during heavy exercise. The miniature nature of human alveolus has been observed as exemplified with 100-300 $\mu$ m diameters in humans [27] though how such value was arrived at is not certain.

Similar observation was made with respect to the result obtained for the female subject (Table $3 \mathrm{~b}$ ). However, the trend in value of alveolar radii with increasing age was less regular than in male, from coronal perspective. The trend was totally similar to male from sagittal perspective. The calculated radii of alveolus for age bracket above 10-19 years for the female (Table 3b) are shorter than the value 1.25 exp $(-4)$ metres [26]. The values calculated in this research coronally and sagittally for the female (Table $3 \mathrm{~b}$ ) are very similar to the average minimum radius in the literature [26]. The older age brackets, male and female, seem to possess longer radii of the alveoli.

Table 3b The various values of alveolar radii for different female age-brackets as a function of trachea length and diameter.

\begin{tabular}{|l|l|l|l|}
\hline Age groups (years) & \multirow{2}{*}{ No. of Subjects } & Radius / exp (-4) m & Radius / exp ( - 4) m \\
\cline { 3 - 4 } & & Coronal perspective & Sagittal perspective \\
\hline $10-19$ & 20 & $0.999 \pm 0.120$ & $1.006 \pm 0.099$ \\
\hline $20-29$ & 98 & $1.086 \pm 0.098$ & $1.079 \pm 0.128$ \\
\hline $30-39$ & 64 & $1.106 \pm 0.134$ & $1.126 \pm 0.171$ \\
\hline $40-49$ & 45 & $1.146 \pm 0.149$ & $1.159 \pm 0.164$ \\
\hline $50-59$ & 65 & $1.139 \pm 0.120$ & $1.173 \pm 0.149$ \\
\hline $60-69$ & 48 & $1.159 \pm 0.129$ & $1.180 \pm 0.170$ \\
\hline $70-79$ & 36 & $1.133 \pm 0.178$ & $1.272 \pm 0.171$ \\
\hline
\end{tabular}

The grouping by age and the number of subjects and the diameter of the trachea are as adopted from the work of Breatnach et al [19]; the arithmetic means of the length, $10.67 \mathrm{~cm}$ and $12.4 \mathrm{~cm}$ were calculated from values adopted from the work of [22].

An important observation is that the radii for the older subjects are longer than for the younger subjects. The data seem to represent what are expected of adolescent and adults in general. This is in view of the fact that it is well known and documented that the neonates and infants possess higher respiratory rate $\left(R_{\mathrm{r}}\right)$ than adolescence and adults (www.healthline.com/health/normal /respiratory rate). There seem to be inverse relation between $R_{\mathrm{r}}$ and the tidal volume (www.healthline.com/health/normal /respiratory rate). It is probable that an $R_{\mathrm{r}}$ equal to 16 may be associated with younger subjects. If so the adoption of $R_{\mathrm{r}}$ equal to 12 in the computation in this research may well represents what is expected of older generation. Credence to this issue can be found in the experimentally backed view that "there were no differences between boys and girls until age 14, when girls' trachea stopped growing. The data suggest that male tracheas continue to enlarge (but not lengthen) for a time after growth in height ceases. Mean transverse diameters tended to be greater than mean AP (anterior-posterior) diameters to the age of 6; the diameters were then nearly identical until age 18, when the AP diameters usually became slightly larger" [22]. Besides, infants have much shorter length and diameter than school-age and adult subjects [22]. Children below school age need exclusive feature research. Though for the sick, the reportage that neonates with respiratory distress syndrome (ROS) typically breathe over 100 times a minute, with a smaller tidal volume justifies the preceding argument (www.sciencedirect.com /topics/respiratory-minute-volume). A tidal volume, $T_{\mathrm{v}} \approx 6-10 \mathrm{~mL} / \mathrm{kg}$ and $4-6 \mathrm{~mL} / \mathrm{kg}$ in the preterm are $\mathrm{known}$, and, $R_{\mathrm{r}}$ value equal to 30 to 60 breaths/minute is also known (www.healthline.com/health/normal /respiratory rate).

The above discussion is penitent in the light of Eq. (8) which when applied would have yielded very high value of alveolar radii for the infants much more than the adults in general because $r_{\text {alv }}$ is inversely proportional $1 / \sqrt[3]{R_{\mathrm{v}}}$. Nonetheless, $r_{\mathrm{alv}}$ is also directly proportional to $\sqrt[3]{l_{\text {trach }}}$ and $\sqrt[27]{r_{\text {trach }}^{26}}$ such that $r_{\text {trach }}$ and $l_{\text {trach }}$ being much longer in adolescence and adults than 0 to preschool age in particular, the effect of the inverse relationship of $r_{\text {alv }}$ with $R_{\mathrm{v}}$ may be drastically attenuated. Once again this is to be investigated separately. 


\section{Conclusion}

Although the rate of gas flow in volume per cross-sectional area of individual tract per unit time increases for awhile and then decreases due to rapidly decreasing volume of gas flowing through the tract per unit time, the total distance covered per unit time is increasingly high making delivery of gas to the alveoli very rapid. The derived equations could be used for the computation of alveolar radius. There are gender, demographically and racially based differences in the radius of alveolus. Information regarding the rate of gas flow through the cylinder-like respiratory tract and the number of functional tracts per generation could enhance the evaluation of the efficacy and effectiveness of therapeutic measures. The equation derived in this research can be applied to effort directed to the determination of alveoli radii in infants and pre-school age in general. The model may be modified in order to determine the radii of alveoli during physical exercise as part of feature research.

\section{Compliance with ethical standards}

\section{Acknowledgments}

The supply of electric power by the Management Royal Court Yard Hotel Agbor (in Delta State, Nigeria) during the preparation of the manuscript is always deeply appreciated.

\section{Dedication}

This paper is dedicated to Late Madam Katherine Johnson who was hardly mentioned due to, perhaps, deep discrimination, until recently by a former president, despite her tremendous contribution to American space programme, which took astronauts to, and most importantly, back to the Earth to tell the full story.

\section{Disclosure of conflict of interest}

The author has declared no competing interest.

\section{References}

[1] Kjærgaard T, Cvancarova M, Steinsva SK. Relation of nasal air flow to nasal cavity dimensions. Arch Otolaryngol Head Neck Surg. 2009; 135 (6): 565-570.

[2] Leong AFT, Buckley GA, Paganin DM, Hooper SB, Wallace MJ, Kitchen MJ. Real-time measurement of alveolar size and population using phase contrast x-ray imaging. v. Biomed. Opt. Express. 2014; 5(11): 1-15.

[3] Brown HT, Escombe F. Static diffusion of gases and liquids in relation to the assimilation of carbon and translocation in plants. Philos. Trans. R. Soc. B. 1900; 193: 223-291.

[4] Iorungwa IS. A mathematical model of airflow into the lungs and its subsequent diffusion into the blood stream. Thesis. 2011.

[5] Udema II. Reason for higher rate of gas flow per unit cross-sectional area of smaller pore aperture. Asian J. Phys. Chem. Sci. 2018; 6(3): 1-18.

[6] Schriever VA, Hummel T, Johan N. Lundström JN, Freiherr J. Size of nostril opening as a measure of intranasal volume. Physiol. Behav. 2012; 110-111 (2013): 3-5.

[7] Patwa A, Shah A. Anatomy and physiology of respiratory system relevant to anaesthesia. Indian J. Anaesth. 2015; 59 (9): 533-541.

[8] Weibel ER. Morphometry of the human lung. Springer Verlag and Academic Press. New York. 1963.

[9] Gehr P. Annexe A. Anatomy and morphology of the respiratory tract. The report of a task group of committee 2. 1984; 24 (1-3): 121-166.

[10] Udema II. A novel mathematical equation for a noninvasive determination of the number of alveoli of the human lung. Asian J. Biol. 2020; 10 (1): 38-47.

[11] Zaidi AA, Mattern BC, Claes P, McEcoy B, Hughes C, Shriver MD. Investigating the case of human nose shape and climate adaptation. PLoS Genet. 2017; 13(3): 1-31.

[12] Hsia CCW, Hyde DM, Weibel ER. Lung structure and intrinsic challenges of gas exchange. Compr. Physiol, 2016; 6 (2): 827-895. 
[13] Coyne K, Caretti D, Scott W, Johnson A, Koh F. Inspiratory flow rates during hard work when breathing through different respirator inhalation and exhalation resistances. J. Occup. Environ. Hyg. 2006; 3(9): 490-500.

[14] Hlastala MP, Powell FL, Anderson JC. Airway exchange of highly soluble gases. Appl Physiol. 2014; 114: 675-680.

[15] Fowler WS. Lung function studies II. The respiratory dead space. Am J. Physiol. 1948; 154: 405-416.

[16] Hildebrandt J, Structural and mechanical aspects of respiration. In: Textbook of Physiology, edited by H. D. Patton, A. F. Fuchs, B. Hille, A. M. Scher, and R. Steiner. Philadelphia, PA: Saunders. 1989.

[17] George SC, Babb AL, Hlastala MP. Dynamics of soluble gas exchange in the airways iii. Single-exhalation breathing maneuvers. The American Physiological Society. 1993; 2439-2449.

[18] Yeh HC, Schum GM. Models of human lung airways and their application to inhaled particle deposition. Bull. Math. Biol. 1980; 42: 46I-480.

[19] Breatnach E, Abbott GC, Fraser RG. Dimensions of the normal human trachea. ATR. 1984; 141, 903-906.

[20] Zahhedi-Nejad N, Bakhshayesh-Karam M, Kahkoei S. Normal dimension of trachea and two main bronchi. Pol. J. Radiol. 2011; 76(4): 28-31.

[21] Lee JW, Son J-S, Choi J-W, Han Y-J, Lee J-R. The comparison of the length and diameters of main bronchi measured from two- dimensional and three- dimensional images from the same patients. Korean J. Anesthesiol. $2014 ; 66$ (3): 189-194.

[22] Griscom NT, WohI MEN. Dimensions of the growing trachea related to age and gender. AJR. 1986; 146: $233-237$.

[23] Mi W, Zhang C, Wang H, Cao J, Li C, Yang L. et al. Measurement and analysis of the tracheobronchial tree in Chinese population using computed tomography. PLosOne. 2015; 10 (4): 1-14.

[24] Phalen RF, OIdham MJ, Beaucage CB, Crocker TT, Mortensen JD. Postnatal enlargement of human trachcohronchial airways and implications for particle deposition. Anat. Rec. 1985; 212: 368-380.

[25] Cinar U, Halezerogglu S, Okur E, Inanici M, Kayaoglu S. Tracheal length in adult human: The results of 100 autopsies. Int. J. Morphol. 2016; 34 (1): 232-236.

[26] Balásházy I, Hofmann W, Farkas A, Madax BG. Three-dimensional model for aerosol transport and deposition in expanding and contracting alveoli. Inhal. Toxicol. 2008; (16): 611-621.

[27] Namati E, Thiesse T, de Ryk J, McLennan G. "Alveolar dynamics during respiration: are the pores of Kohn. A pathway to recruitment?” Am. J. Respir. Cell Mol. Biol. 2008; 38(5): 572-578.

\section{Author's short biography}

Udema Iloh Ikechukwu (http://orcid.org/0000-0001-5662-4232)
My interest covers mainly subjects such as biochemistry, biophysics (and related field) and very
limited extra-curricular subject such as atomic and nuclear physics. My general interest includes
field of science amenable to basic mathematics. My recent interest in respiratory physiology is due
to the challenge imposed by the global pandemic which compromised respiratory system to an
extremely dangerous proportion. My highest degree is a Ph.D in Biochemistry.

